\title{
TWO NOTES ON METRIC GEOMETRY
}

\author{
RALPH ALEXANDER
}

\begin{abstract}
If $\mu, \int d \mu=1$, is a signed Borel measure on the unit ball in $E^{3}$, it is shown that $\sup _{\mu} \iint|p-q| d \mu(p) d \mu(q)=2$ with no extremal measure existing. Also, a class of simplices which generalizes the notion of acute triangle is studied. The results are applied to prove inequalities for determinants of the Cayley-Menger type.
\end{abstract}

1. A counterexample. In this article $\mu$ will always denote a signed Borel measure, concentrated on a compact set $K$ in Euclidean space, which satisfies $\int d \mu=1$. Let $I(\mu)$ denote the integral $\iint|p-q| d \mu(p) d \mu(q)$. It was shown in [1] and [2] that if $K \subset E^{n}$, then there is a constant $b_{n}$ such that $\sup _{\mu} I(\mu)<$ $b_{n}$ (diameter of $K$ ). It was conjectured that an extremal measure $\mu_{0}$ would always exist so that $I\left(\mu_{0}\right)=\sup _{\mu} I(\mu)$. This conjecture was known to be true in case $K$ was a finite set or the surface of a ball.

However, we now show that an integral formula developed in [2], together with Archimedes' beautiful theorem on zonal areas, allows us to construct a counterexample when $K$ is a solid ball in $E^{3}$.

Let us assume that the ball $K$ has radius 1 , and let $N$ be a large positive number. Place a uniform measure of total mass $N$ on the surface of $K$, and place a uniform negative measure of total mass $1-N$ on the surface of the concentric ball of radius $1-N^{-1}$. The measure $\mu_{N}$ is defined to be the sum of these two uniform measures.

A key observation is that if $t_{1}$ and $t_{2}$ are two parallel planes which both cut the ball of radius $1-N^{-1}$, then $\mu_{N}$ assigns measure zero to the portion of $K$ between $t_{1}$ and $t_{2}$. This follows from Archimedes' theorem.

Let $\tau$ be the usual invariant measure on the planes of $E^{3}$ such that the measure of the set of planes which cut a line segment is the length of the segment. We shall use without proof the fact that the measure of the set of planes in $E^{3}$ which cut a ball of radius $R$ is $4 R$.

If $t$ is a plane which cuts $K$, let $A_{t}$ and $B_{t}$ be the portions of $K$ lying in the respective open half-spaces determined by $t$. In [2] it was established that

$$
I(\mu)=2 \int \mu\left(A_{t}\right) \mu\left(B_{t}\right) d \tau(t) .
$$

Also, it was shown that for almost all $t, \mu\left(A_{t}\right)+\mu\left(B_{t}\right)=1$, and hence for almost all $t, \mu\left(A_{t}\right) \mu\left(B_{t}\right) \leqslant \frac{1}{4}$.

Received by the editors September 20, 1976.

AMS (MOS) subject classifications (1970). Primary 52A50.

Key words and phrases. Metric, invariant measure, inversion, Cayley-Menger determinant.

○ American Mathematical Society 1977 
LEMMA 1. There is no signed Borel measure of total mass 1, concentrated on the unit ball $K$, for which $\mu\left(A_{t}\right)=\mu\left(B_{t}\right)=\frac{1}{2}$ for almost all $t$.

Proof. Suppose such a measure exists. Let a plane $t_{0}$ cut $K$ so that the volume of $A_{t_{0}}$ is $\varepsilon>0$. The set of planes $t$ for which $A_{t} \subset A_{t_{0}}$ has positive $\tau$-measure, and hence $\mu\left(A_{t}\right)=\frac{1}{2}$ for at least one such $t$. Thus $|\mu|\left(A_{t_{0}}\right) \geqslant \frac{1}{2}$. Since this is true for any $\varepsilon>0$, we quickly deduce by measure continuity from above that each point on the surface of $K$ is an atom of $|\mu|$-measure at least $\frac{1}{2}$. This cannot be true since $|\mu|(K)<\infty$ by the definition of signed measure. (See the book of Halmos [5, Chapter 6].)

The lemma together with (1) allows us to conclude that if $\mu_{0}$ exists then $I\left(\mu_{0}\right)<2 \cdot 4 \cdot \frac{1}{4}=2$. The measure of the planes $t$ which cut the ball of radius $1-N^{-1}$ is $4\left(1-N^{-1}\right)$ and for any such $t, \mu_{N}\left(A_{t}\right) \mu_{N}\left(B_{t}\right)=\frac{1}{4}$ by Archimedes' theorem. If $t$ is a plane which does not cut the ball of radius $1-N^{-1}$, then certainly $\mu\left(A_{t}\right) \mu\left(B_{t}\right) \geqslant 0$. Thus

$$
I\left(\mu_{N}\right) \geqslant 2\left[4\left(1-N^{-1}\right)\right] / 4=2\left(1-N^{-1}\right) \text {. }
$$

By choosing $N$ large enough, $I\left(\mu_{N}\right)>I\left(\mu_{0}\right)$, a contradiction. We have proved the following theorem.

THeOREM 1. Let $K$ be the unit ball in $E^{3}$. Then $\sup _{\mu} I(\mu)=2$, but no extremal measure $\mu_{0}$ exists.

We remark that the same type of theorem can be proved when $K$ is allowed to range over the family of sets of constant width 2 in $E^{3}$. Thus if $K$ is any compact set of diameter not exceeding 2 and $\mu$ is concentrated on $K, I(\mu)<$ 2 , and this is the best possible. The corresponding question for positive measure is open. The paper of Björck [3] contains much information about $I(\mu)$, restricted to the class of positive measures.

2. Acute simplices and Cayley-Menger determinants. If $K$ is a finite set in Euclidean space, the functional $I(\mu)$ achieves an absolute maximum for a unique, necessarily atomic, measure $\mu_{0}$ as was shown in [1]. Also, it was shown that

$$
I\left(\mu_{0}\right)=2 R^{2}
$$

where $R$ is the radius of the least sphere which circumscribes the metric space $\left(K, d^{1 / 2}\right)$ viewed as a subset of Euclidean space. The work of I. J. Schoenberg [6] assures us that if $d$ is the Euclidean metric, then $\left(K, d^{1 / 2}\right)$ embeds isometrically into $E^{m}$ as the vertices of a nondegenerate $m$-simplex if $|K|=m$ +1 . The number $R$ is the radius of the $(m-1)$-sphere which contains the points of $K$.

Definition 1 . We call a simplex in $E^{m}$ with distinct vertices $p_{0}, p_{1}, \ldots, p_{m}$ $r$-acute if there exists a simplex in $E^{r}$ with vertices $q_{0}, q_{1}, \ldots, q_{m}$ such that $\left|p_{i}-p_{j}\right|^{2}=\left|q_{i}-q_{j}\right|$ for all $i, j$.

The number $r$ is not unique, but generally the least possible value would be chosen. The previously mentioned work of Schoenberg assures us that the 
acute simplex of the definition must be a nondegenerate $m$-simplex. Our next theorem says much more. Before stating the theorem we point out the obvious fact that the altitude from a vertex $p_{i}$ of a simplex does not exceed $\min _{j}\left|p_{i}-p_{j}\right|, i \neq j$.

THEOREM 2. Let $S$ be an r-acute simplex. Then there is an absolute positive constant $c_{r}$ such that the altitude from any vertex $p_{i}$ is not less than

$$
c_{r} \min _{j}\left|p_{i}-p_{j}\right|, \quad i \neq j
$$

LEMMA 2. Let $p_{1}^{\prime}, \ldots, p_{m}^{\prime}$ be the images of $p_{1}, \ldots, p_{m}$ under the inversion through a sphere of radius 1 centered at $p_{0}$. Then $p_{0}^{\prime}, p_{1}^{\prime}, \ldots, p_{m}^{\prime}\left(p_{0}^{\prime}=p_{0}\right)$ are the vertices of an $r$-acute simplex $S^{\prime}$ if and only if $p_{0}, \ldots, p_{m}$ are the vertices of an r-acute simplex $S$.

Proof. Let $q_{0}, \ldots, q_{m}$ be points in $E^{r}$ for which $\left|q_{i}-q_{j}\right|=\left|p_{i}-p_{j}\right|^{2}$. First suppose that neither $i$ nor $j$ is 0 , and that $q_{0}^{\prime}, q_{1}^{\prime}, \ldots, q_{m}^{\prime}$ are the respective images of $q_{0}, \ldots, q_{m}$ under the inversion through the unit sphere centered at $q_{0}=q_{0}^{\prime}$. We note that

$$
\left|q_{i}^{\prime}-q_{j}^{\prime}\right|=\left|q_{i}-q_{j}\right|\left[\left|q_{i}-q_{0}\right|\left|q_{j}-q_{0}\right|\right]^{-1}=\left|p_{i}^{\prime}-p_{j}^{\prime}\right|^{2} \text {. }
$$

Also $\left|q_{0}^{\prime}-q_{i}^{\prime}\right|=\left|q_{0}-q_{i}\right|^{-1}=\left|p_{0}^{\prime}-p_{i}^{\prime}\right|^{2}$.

The proof in the opposite direction is symmetrical.

Proof of Theorem 2. Using the notation of Lemma 2, we see that the altitude $h$ from $p_{0}$ in the simplex $S$ is precisely $D^{-1}$ where $D$ is the diameter of the circumsphere (in $E^{m}$ ) of the simplex $S^{\prime}$. This follows at once from the fact that the inversion at $p_{0}$ sends the hyperplane spanned by $p_{1}, \ldots, p_{m}$ into a sphere which contains $p_{0}$, and that the point in the hyperplane nearest to $p_{0}$ will be sent to a point diametrically opposite to $p_{0}$ on the circumsphere of $S^{\prime}$.

Next we use known results about the functional $I(\mu)$ to obtain an upper bound on $D$, which will give a lower bound on $h=D^{-1}$. The measure $\mu$ will be defined by $\mu\left(q_{i}^{\prime}\right)=x_{i}$ with $\sum x_{i}=1$ so that

$$
I(\mu)=\sum\left|q_{i}^{\prime}-q_{j}^{\prime}\right| x_{i} x_{j}=\sum\left|p_{i}^{\prime}-p_{j}^{\prime}\right|^{2} x_{i} x_{j} .
$$

We know that there is an absolute constant $b_{r}$ such that

$$
I(\mu) \leqslant b_{r}\left\{\max _{i, j}\left|q_{i}^{\prime}-q_{j}^{\prime}\right|\right\} \quad \text { for any } \mu .
$$

This result is found in [1, Theorem 3.8]. Combining (3) and (2) we obtain

$$
\frac{1}{2} D^{2} \leqslant b_{r}\left\{\max _{i, j}\left|p_{i}^{\prime}-p_{j}^{\prime}\right|^{2}\right\} \text {. }
$$

Since $\max _{i, j}\left|q_{i}^{\prime}-q_{j}^{\prime}\right| \leqslant 2 \max _{i}\left|q_{0}^{\prime}-q_{i}^{\prime}\right|$, we have $D^{2} \leqslant 4 b_{r} \max _{i}\left|p_{0}^{\prime}-p_{i}^{\prime}\right|^{2}$. Since $\left|p_{0}^{\prime}-p_{i}^{\prime}\right|=\left|p_{0}-p_{i}\right|^{-1}$, it follows that $D^{-1}=h \geqslant c_{r} \min _{i}\left|p_{0}-p_{i}\right|$ where $c_{r}=\left(2 b_{r}^{1 / 2}\right)^{-1}$. This completes the proof. It may be true that $c_{r}$ $=\sqrt{2} / 2$ will work in all dimensions.

As an application of the theorem we prove an inequality for CayleyMenger type determinants. 
Corollary 1. Let $q_{0}, q_{1}, \ldots, q_{n}$ be points in a Euclidean space, then there is an absolute positive constant $d_{n}$ such that

$$
\left|\operatorname{det}\left(\begin{array}{cc}
0 & 1 \\
1 & \left|q_{i}-q_{j}\right|
\end{array}\right)\right| \geqslant d_{n}\left[\min _{i \neq j}\left|q_{i}-q_{j}\right|\right]^{n} .
$$

Proof. We consider an $n$-acute simplex $S$ whose vertices $p_{0}, \ldots, p_{n}$ satisfy $\left|p_{i}-p_{j}\right|^{2}=\left|q_{i}-q_{j}\right|$. Any subsimplex of an acute simplex is certainly an acute simplex. Also, any altitude from any vertex of a subsimplex of $S$ will satisfy $h \geqslant c_{n} \min _{i \neq j}\left|p_{i}-p_{j}\right|$ by Theorem 2. Thus $\operatorname{Vol}(S)$ is at least $(n !)^{-1}\left[c_{n} \min _{i \neq j}\left|p_{i}-p_{j}\right|\right]^{n}$.

It is well known (see [4, Chapter 4]) that

$$
\left|\operatorname{det}\left(\begin{array}{cc}
0 & 1 \\
1 & \left|p_{i}-p_{j}\right|^{2}
\end{array}\right)\right|=d_{n}^{\prime}[\operatorname{Vol}(S)]^{2} \text {. }
$$

Substituting the previously mentioned lower bound for $\operatorname{Vol}(S)$ completes the proof with $d_{n}=d_{n}^{\prime}(n !)^{-2} c_{n}^{2 n}$.

Although the Cayley-Menger type determinant arises most frequently, $\operatorname{det}\left(\left|q_{i}-q_{j}\right|\right)$ is also of interest. We note that this determinant equals

$$
\left(\prod_{i=1}^{n}\left|q_{0}-q_{i}\right|^{2}\right) \operatorname{det}\left(\begin{array}{cc}
0 & 1 \\
1 & \left|q_{i}^{\prime}-q_{j}^{\prime}\right|
\end{array}\right)
$$

where $\left|q_{i}^{\prime}-q_{j}^{\prime}\right|$ are the inverted distances previously discussed. Thus, if need be, we can also bound this determinant away from zero.

In closing, we note that inequality (3) can also be easily derived from formula (1), letting $K=\left\{q_{0}^{\prime}, \ldots, q_{m}^{\prime}\right\}$. We would have, using the fact that any set of diameter $D$ is contained in a ball of radius $D$,

$$
I(\mu) \leqslant 2\left(\frac{1}{4}\right)(\text { diameter } K) \tau_{r},
$$

where $\tau_{r}$ is the measure of the hyperplanes in $E^{r}$ which cut a unit ball in $E^{r}$.

\section{REFERENCES}

1. R. Alexander and K. B. Stolarsky, Extremal problems of distance goemetry related to energy integrals, Trans. Amer. Math. Soc. 193 (1974), 1-31. MR 50\# 3121.

2. R. Alexander, Generalized sums of distances, Pacific J. Math. 56 (1975), 297-304.

3. G. Björck, Distributions of positive mass, which maximize a certain generalized energy integral, Ark. Mat. 3 (1956), 255-269. MR 17, 1198.

4. L. M. Blumenthal, Theory and applications of distance geometry, Clarendon Press, Oxford, 1953. MR 14, 1009.

5. P. R. Halmos, Measure theory, Van Nostrand, Princeton, N. J., 1950. MR 11, 504.

6. I. J. Schoenberg, On certain metric spaces arising from Euclidean spaces by change of metric and their imbedding in Hilbert space, Ann. of Math. (2) 38 (1937), 787-793. 